The circumstances under which nurses have been admitted suffering from typhus fever have been those where their patient has been treated for an unrecognized attack, and unusually free admission of fresh air and other necessary precautions have not, therefore, been adopted. We can recall several such instances, notably two cases of supposed pneumonia which resulted in the admission of three and four nurses respectively with typhus, the attack in all cases being severe. Such occurrences go to prove not only the value of fresh air as a diluent of typhus contagion, but also its virulence at close quarters.

It is the duty of the hospital porters to carry patients from the ambulance to the wards, and within our experience none of these men have contracted typhus fever. Their contact is of short duration and not very intimate, so that the opportunity for contracting the disease may be slight. The case of the nurses is different, as they have to wash the patients and remove their clothing.

At Aberdeen typhus has occurred only among those who have assisted in removal and cleansing of patients, those nurses not so engaged having escaped infection. This has led Dr. Hay to regard fleas with suspicion as possible carriers of infection, but though they are in abundant evidence among the Liverpool cases the nurses have, within our experience, enjoyed immunity from typhus. They express some surprise, as a matter of fact, that they are seldom bitten by fleas, but it is hardly likely that the experience of nurses in the two cities differs greatly in this respect.

It is interesting to note also the experience of the nursing staff on another point relating to immunity, namely, the possibility of the protection by repeated small doses of the contagion, probably in a diluted condition. It has been thought that repeated headaches among those attending on typhus patients are an indication of such diluted dosage. Frequent frontal headache is complained of by nurses during their spell of typhus duty, which is now limited to a few weeks for this reason. At the same time it should be noted that the bulk of the cases are admitted during the winter months, and that the low temperature of the air, which is freely admitted, might possibly contribute to cause this. The nurses state, however, that the headache is less frequent during the coldest weather.

In conclusion, we wish to express our indebtedness to $\mathrm{Dr}$. R. I. Richardson, Visiting Physician to the City Hospital North,.Liverpool, for his kind permission to make use of the cases in hospital for the purposes of this paper.

\section{SOME EXPERIMENTS IN THE TREATMENT OF TRYPANOSOMIASIS.}

By H. WOLFERSTAN THOMA8, M.D., C.M.McGirL,

J. L. Todd Memorial Fellow in Tropical Medicine, the Liverpool School of Tropical Medicine, Johnston Tropical Laboratory,

$$
\text { University, Liverpool. }
$$

THe discovery of a trypanosome affecting man by Dutton, ${ }^{1}$ and, quickly following upon this, the finding by the Royal Dociety's Commission ${ }^{2}$ that trypanosomes play such an important rôle in sleeping sickness, has stimulated the efforts made to find some drug which would act on the trypanosomata as quinine does on the plasmodium of malaria.

Trypanosomiasis has for many years engaged the serious attention of the veterinary profession; the heavy loss amongst horses and cattle in tropical countries, from various trypanosomic diseases naturally caused the trial of many drags. Of all the drugs tried arsenic is the only one which seems to have given favourable results ; reports concur that it mitigates the disease, and in some cases cures have been recorded.

Laveran, ${ }^{3}$ working experimentally on trypanosomiasis, published in 1902 his results on the treatment by means of sodium arseniate of rats infected with the nagana parasite (T. brucei) Since that time he has published several papers on arsenic in the treatment of various experimental trypanosomic diseases. Moore ${ }^{4}$ and Chichester ${ }^{5}$ record their results on the treatment of cattle in Southern Nigeria with sodium arseniate. Broden and others have treated cases of human trypanosomiasis with arsenic, usually in the form of Fowler's solution, with very encouraging results.

Another drug which in the last year was introduced by Ehrlich and Shiga ${ }^{7}$ is trypanroth. The authors treated rats and other animals infected with the trypanosoma of mal de Caderas (T. equinum). Their results are most encouraging.
Laveran has since then recorded his results with the treatment of trypan red in various trypanosomic diseases, and more recently on mixed treatment with trypan red and sodium arseniate. Wendelstadt ${ }^{8}$ has reported on the use of malachite green. His results are not very successful, and the dangers of producing necrosis are too great to warrant its use as 8 therapeutical agent.

Arsenic in the treatment of the various pathogenic trypanosomic diseases in animals seems rather to mitigate the disease, to cause the parasite to apparently disappear from the animal's blood, and to prolong the life of the animal. Unfortunately the usual history is that if treatment be discontinued, even after a prolonged course of the drug, the animal after a varying length of time once more exhibits the symptoms, and finally succumbs to the disease. Undoubtedly some of the more resistant animals do recover. Laveran and Mesnil in their classic work, Trypanosomes et Trypanosomiasis, from their numerous experiments conclude that the administration of arsenic kills the trypanosomes which are free in the blood, but when once the drug is eliminated or fixed in the tissues the surviving parasites commence to multiply and the organisms once more reappear in the peripheral circulation unless another injection of arsenic be given. On the administration of a second dose, the parasites disappear only to reappear, and even though treatment be kept up, the majority of the animals succumb either from the disease or from the toxic effects of the drug. Some of their animals, rats and dogs, have been cured. Laveran records the extensive necrosis and the pain which is apt to follow the administration of arsenious acid.

In 1903, while at McGill University, I repeated some of the experiments with arsenic on animals infected with the nagana parasite. On my taking up trypanosomiasis research in the Liverpool School of Tropical Medicine, I started a series of experiments with sodium arseniate. My experiences were the same as Laveran's. Many of my rats either died from the disease, were killed by the drug, or succumbed to an extensive ulceration around the site of injection.

On the publication of Ehrlich and Shiga's result with trypan red, a series of animals infected with different trypanosomes were treated with the dye. The best results were obtained with mal de Caderas infected animals. The results were not so good with animals infected with surra and nagana parasites. The results were still worse with dourine, Gambian horse, and T. gambiense strains. The parasite disappeared for a few days, to reappear, and the duration of the disease was not greatly prolonged. A combination of trypan red and arsenic seemed to be worth trying; the results obtained were more promising, but unfortunately trypan red also caused a nephritis, and by its chemiotaxic properties very extensive necroses sometimes resulted. On monkeys especially, the subcutaneous injection of the dye, either alone or in eombination with arsenious acid, induced ulceration, which so undermined the health of the animals that they succumbed to any outbreak of disease, which occurred only too frequently amongst my numerous animals. It was these untoward accidents which induced me to seek a preparation of arsenic less toxic, and the subcutaneous injection of which entailed less danger of necrosis. An aniline compound, metaarsensaureanilid (atoxyl). having the formula $\mathrm{C}_{6} \mathrm{H}_{6} \mathrm{NO}_{2} \mathrm{As}$, attracted my attention. This preparation has been before the profession since 1900, and various workers have recorded its worth in the treatment of various skin affections and in anaemia. It contains 37.69 per cent. arsenic, and is claimed to be 40 times less toxic than liquor Fowleri. It is especially suitable for either subcutaneous or intravenous administration. It produces no necrosis, no pain, and very much higher doses of arsenic can be given without producing toxic results. (I have tried the drug in high doses intravenously on myself without ill effects.)

Tentative experiments on rats and rabbits appeared sofavourable that I decided to institute a series of experiments. These observations have been in progress for the last ten months, and have on the whole been most promising. In all $m y$ therapeutic experiments certain conditions were laid down to be followed out:

r. That the animal should be well infected and the presence of parasites determinable in its blood.

2. That the disesse should have been established some time (o cannot expect to treat either man or beast suffering from the disease $n$ the very early stages when no definite symptoms are manifested

3. That some symptoms besides the presence of parasites should be in evidence.
(b) Loss of weight. 
If the treatment of such infected laboratory animals be successful, then such s line of medication ought to be of servioe in the practical treatment of the disease.

\section{Trypanosomes.}

The trypanosomes experimented with are the following:

Trypanosoma Gambiense-

(a) Gambian fever strain (Gunjur)

(b) Congo Free State fever strain.

(c) Uganda sleeping sickness.

(d) Congo Free State sleeping sickness.

* (e) A highly virulent strain derived from one of my cases of sleeping sickness which had only been passed through a monkey, baboon, and a rabbit.

Trypanosoma brucei (nagana).

Trypanosoma evansi (surra)

Trypanosoma equinum (mal de Caderas)

Trypanosoma equiperdum (dourine).

Trypanosoma dimorphum (Gamblan horse disease).

Animals Used.

T. gambiense.-Monkeys, dogs, pups, kittens, rabbits, guinea-pigs, cats and mice.

T. evansi. $\dagger-R a b b i t s$, guinea-pigs, dogs, rats, mice.

T. brucei. $\dagger$-Rabbits, guinea-pigs, rats, mice.

T. equinum.--Dogs, rabbits, guinea-pigs, rats and mice.

T. equiperdum.-Pups.

T. dimorphum.-Dogs, pups, rabbits, guinea-pigs, rats, and mice.

It is not my intention to give a series of experiments in detail, these will appear in the forthcoming memoir of the Liverpool School of Tropical Medicine. All the animals ful filled the conditions laid down, and all contained parasites in their blood. The lowest number was 6 to ro trypanosomes to a field in rabbits infected with the Gambian horse disease, and pups with dourine : up to 50,80 , to 120 or more to a field being present in the animals infected with the very virulent strain called T. gambiense (e)-monkeys, guinea-pigs, dogs, rabbits, kittens, rats-and with surra, nagana and Caderasrats, mice, guinea-pigs.

I have found from experiments that the dosage which the average animal will stand is :

Rabbits (r,700 to 2,000 grams) r.o c.cm. ro per cent. solution.

Monkeys (rhesus) (2,400 grams) x.0 to $x .5 \mathrm{ocm}$. xo per cent. solution. Guinea-pigs $+(600$ grams) 0.3 to 0.5 to $1.0 \mathrm{c.cm}$. 5 per cent. solution. Rats (1 30 grams) 0.5 to 0.8 c.cra. 5 per cent. solution.

Mice (20 to 25 grams) $0 . x$ to 0.2 c.cm. 5 per cent. solution.

These doses are well tolerated by the animals unless almost moribund, in which case it is advisable to give half to three quarters of the dose. With dogs, pups and kittens I have had deas favourable results. The animals appear to exhibit a greater tendency to toxicity when once well infected, and the dose which the trial animals stood will set up serious symptoms in the infected ones.

In my earlier experiments, after the initial injection I waited two days and then administered half the starting dose three times a week, then gradually increasing until I attained the initial dose, and going higher if necessary. I have since tried the effect of giving the initial dose, and then administering once a week the same amount, even increasing it gradually until the animal received one and a half times as much as the primary dose without symptoms. As I have devoted more attention to the treatment of animals infected with the human strains, I have felt it was better to adopt a more rational mode of procedure, and to administer the drug regularly over a period of one, two, or three months rather than inject only when parasites reappeared in the blood. I have applied the same mode of procedure in treating animals infected with the other strains of the trypanosomes. The results are that after a course of treatment the animal survives, whilst its non-treated control dies-parasites are absent from the peripheral circulation, the blood count improves, the animal increases in weight, and the temperature becomes more regular. On injecting the blood of such an animal into a susceptible animal no infection occurs. I have not been content with inoculating small quantities of blood, as from my experience the trypanosomes are very scarce in the blood of treated animals, and for the time being are attenuated,

* So highly virulent that if delay of one or two days occurred in starting treatment the animal would generally die. It is the Gunjur strain $(a)$ and this virulent one $(e)$ which has been chiefly used in my experiments.

$\dagger$ Experiments in progress with a horse infected with surra and a cow infected with nagana parasite.

I Guinea-pigs appear to be more susceptible to toxic effects than the other animals, whilst rats and to a.less extent mice are able to bear with impunity very high doses of the drug; it will be noticed that a rat san be injected with almost the same dose as a guinea-pig four times its woight. Professor Laveran has also noted this with the sodium arseniate-trypan red combined treatment. therefore the injection in small amounts will not serve to infect the subinoculated animal. After treating some of my animals, and assuring myself that parasites were absent, and the general condition being apparently normal, after a period of three months without treatment; I have killed them and injected the whole of their blood into susceptible animals. Such subinoculation controls have not shown parasites in their blood, nor has the blood from such of these proved infective when injected into further control animals; that these animals were not immune was proved by reinoculating them with virulent blood : infection and death took place in the usual time.

The combination of trypan red and arsenic having proved of value, I have combined the dye with atoxyl. The results are satislactory --the treatment does not need to be carried out for so long a period. The only drawback is that animals treated with the combination exhibit a far greater tendency to suffer from nephritis, and at times extensive necrosis occurs at the site of injection.*

On administration of the arsenic compound into an animal showing numerous parasites in the blood, the following action on the trypanosomes will be noted. For the first three and a half to four hours, depending on the dose used, very little change in the parasites can be noted. Between the fourth and tifth hour the effect on the trypanosomes is evident. Some trypanosomes appear to be swollen and their movement is less rapid. If now a series of blood specimens be examined, at intervals of twenty to thirty minutes, the following changes will be seen. The number of slowly-moving trypanosomes increases; many parasites will be seen to be almost motionless. The protoplasm takes on a peculiar groundglass appearance; dark granules appear in the protoplasm; very often a whole series of granules, one behind the other, sometimes in pairs or all clumped together, are seen lying between the macronucleus and the anterior end, or distributed through the whole body of the parasite. At the same time vacuoles are observable, oftentimes being very large. The trypanosomes become deformed, assuming various shapes, the most common being a kite-shaped form with fairly long flagellum, and a tadpole-like one with hardly any free flagellum. These forms especially exhibit greatly impaired movements. At the same time, a noticeable increase of the leucocytes is discernible; phagocytes begin to appear; very often groups of five to seven will be seen.

Up to this time (sixth to seventh hours) the trypanosomes, though decreased, are still present in considerable quantities. Suddenly, in the course of an hour, the number may drop from 40 to 2 or 3 to a field or less; coincident with this is a very marked increase in the number of leucocytes, especially phagocytes. From the ninth to the fourteenth and sixteenth hours the changes are less pronounced and rapid; the trypanosomes gradually disappear. At the eighteenth hour, providing the animal has been injected with the correct amount the parasites are absent from the peripheral circulation, and even though the blood be centrifuged, no parasites can be found.

My colleague, Dr. Breinl and myself, have observed a large number of experiments, examining the animals every one and $a$ half hours for four and $a$ half hours, then every half hour until the parasites were almost absent, and continuing the observations hourly until the twenty-fourth to thirty-second hour after starting the experiment. From a series of these observations we have determined that in hardly any of the forty-six continuously observed animals were paragites to be found after the eighteenth hour. Should, however, the drug be given in smaller amounts the process takes longer, lasting from thirty-six to forty-eight hours. $t$

Phagocytosis has been observed on three occasions; we have witnessed the engulfing of a trypanosome still alive, though almost motionless, and on the other occasions the ingesting of dead trypanosomes.

Observations on the effect of trypan red on the trypanosomes show that almost the same changes take place, though the process is somewhat slower, usually requiring forty-eight hours or more. Leucocytosis appears to be very marked in animals treated with this drug. From the marked leucocytosis which we observed in the course of our experiments, we

* Since I have adopted the intramuscular injections of the dye.I have had very few cases with necrosis.

† Blood films were made before beginning the experiment and at every examination. The results of the examination of these stained films bear out the observations on the fresh blood films. The significance of the
granules seen and the phagocytosis will be discussed in our fuller report. 
decided to cause a hyperleucocytosis by the injection of some leucocycotic agent. The injection of the agent alone caused a hyperleucocytosis but no appreciable diminution of the parasites. When combined with atoxpl, trypan red, or atoxyl-trypan red the blood counts showed very little, if any, increase in the leucocyte counts as compared with any of the three methods of treatment. Later experiments show that some diminution in the number of parasites does occur.

Experiments in Progress.

The following experiments may be of interest :

T. Gambiense (Gunjur Strain).

Rabbit, weight 2,010 grams, parasites appeared on twelfth day, high numbers on forty-sixth day. September $x_{3}$ th-weight $x, 890 \mathrm{grams}$. Blood count-reds 4,980,000, whites 8,860 , haemoglobin 67 per cont. Treatment-1.0 6.cm. 5 per cent. solution three times a week in increasing doses. December 17 th-treatment discontinued. January 4th-weight 2,000 grams. Blood count-reds $6,64^{\circ}, 000$, whites 6,200 , haemoglobin 88 per cent. Between that date and January 13th the animal was in excellent health. Its blood in large doses was non-infective. On January $x^{\text {th }}$ it was noticed to be ailing and to exhibit symptoms of tenesmus. January i 7 th-bled, and whole of blood injected intraperitoneally into a monkey. Up to date this animal remains uninfected. Post mortem.-Very severe haemorrhagic cystitis, the bladder and parts being almost gangrenous-cause unknown. Acute septic peritonitis, especially around bladder. Spleen and glands small, no evidences of a trypanosome infection. Microscopically the spleen showed slight increase in the connective tissue, but no sign of congestion. Kidneys and liver normal.

Rabbit inoculated October $26 \mathrm{th}$, weight 1,860 grams. Blood count-reds 6,620,000, whites 6,700, haemoglobin 89 per cont. Parasites seen November 8th. From that date to January roth trypanosomes always present, but usually scanty. The numbers then rose to 18 to 20 to a feld. Animal had steadily lost weight, and exhibited a profound anaemia. January ioth-weight 1,540 grams. Blood count-reds anaemia. January roth-weight 1,540 grams. Blood count-reds venously $0.8 \mathrm{c.cm}$. 5 per cent. solution. Parasites absent at end of eighteenth hour. Treatment $0.5 \mathrm{c.cm}$., later $1.0 \mathrm{c.cm}$., until $2.0 \mathrm{c.cm}$ of 5 per cent. solution administered twice a week. Weight at present r,980 grams. Blood non-infective. Blood count-reds 6,415,000, whites 6,600 , haemoglobin 82 per cent.

Guinea-pig infected for two months, weight 6ro grams; parasites 26 to 40 to a field. Received 0.3 6.cm. Io per cent. solution.' Parasites absent after fifteenth hour. Treatment, $0.3 \mathrm{com}$. of 5 per cent. solution three times a week for two months. Weight 730 grams. Treatmen discontinued. Sixty-two days later animal died from infective bronchopneumonia. Whole blood inoculated into three rats, which have remained uninfected for forty days.

$$
\text { Stratn " } E \text {." }
$$

Rhesus, weight 2,815 grams, inoculated November 16 th. Blood coun -reds 5,220,000, whites 12,600 , haemoglobin 78 per cent. Positive November $218 \mathrm{st}$. On November $23 \mathrm{rd} 12$ to 17 to a field; weight 2,420 grams. Blood count-reds 4,600,000, whites 7,200, haemoglobin 73 per cent. Parasites 100, 00 per c.mm. Oedema of eyelids and bridge of nose. Received $0.8 \mathrm{~cm}$. ro per cent. solution subcutaneously. Four hours later reds $4,450,000$, whites 24,800 ; parasites 40,000 per c.mm. At eighth hour reds $4.740,000$, whites 25,200 , parasites $x$ to 89 flelds. At twenty-fourth hour reds $5,050,000$, whites 46,000 . Blood negative; the eucocyte count remained high for two days and then fell. Parasites continued absent. Treatment x.o c.cm. of ro per cent. solution twice a week. The animal increased in weight, the erythrocytes and haemoglobin rose. On the thirty-ninth day the animal developed dysentery, and died on the forty-eighth day. Post mortem.-Very severe haemorrhagio and necrotic enteritis. Spleen slightly enlarged, glands small ; inguinal group haemorrhagic. Kidneys normal. Blood non-infective in amoun of $x .0$ c.om., but infective if 15 c.cm. of pure blood was used.

$$
\text { Trypanosoma Brucei. }
$$

Guinea-pig inoculated August ist, weight 5 ro grams. October 29th weight 340 grams. Parasites 40 to 60 to a fleld. Received $0.4 \mathrm{c.cm}$. per cent. solution. Parasites absent at nineteenth hour. Treatment, $0.4 \mathrm{c.cm}$. 5 per cent. solution three times a week for $2 \frac{1}{2}$ months. Treatment discontinued, February 28 th, weight 560 grams ; animal killed and three rats inoculated with whole blood; animals negative. Non-treated guinea-pigs have lived on an average forty to forty-five days.

Rats infected with nagana were treated two, three, and four days after parasites appeared, when in the last stage of the disease and showing the semi-comatose condition which is so often seen. Rat, Exp. 72, weight 135 grams, parasites 200 or more to a field, received o $5 \mathrm{c.cm}$. Of 5 per cent. solution. Parasites absent at twentieth hour. Treated regularly twice a week, dose $0.3 \mathrm{c.cm}$. 5 per cent. solution; parasites never seen. Some have lived as long as 102 days, when they died from epidemics of bronchopneumonia or dysentery. Subinoculated rats remained negative. In rats not treated after the initial dose parasites appeared in seven to eleven days, but if treatment was then begun the parasites disappeared. Increase of weight and percentage of haemoglobin noticed. T. Evansi and T. equinum Animals.-Same history

T. equiperdum experiments in progress, apparently good results.

T. Dimorphum

With this parasite the results have not been 80 good. The parasites disappear usually in eighteen to twenty-four hours after injection, very degenerated forms are seen, but after a period of sixteen to thirty days they are apt to reappear, and very much higher doses have to be given to cause them to disappear. No apparent recovery can be recorded. Undoubtedly the regular administration of this drug has retarded the disease, and the animals have outlived their controls by many months, but the favourable signs - increase of weight, regular temperature, and improvement in the blood counts are wanting. Laveran also records. the greater resistance of animals infected with this parasite to the sodium arseniate treatment.

Trypan Red*-Atoxyl Combinalion.

Rabbit infected with virulent strain " $e$," T. gambiense; weight, 2,450 grams. Parasites 80 to field. I c.cm. ro per cent. atoxyl intravenously; thirty-six to seventy-two hours later 8 to $12 \mathrm{c.cm}$. of I per cent. trypan red subcutaneously. Treatment once every six days. Parasites have disappeared, increase of weight; the anaemis is still present, though considerably lessened. After the sixth treatment the nephritis. One had to be killed because of extensive sloughing around injection area, its subinoculated animals are negative.

Gambian Horse.

Better results are to be recorded with the combined treatment. The trypanosomes are absent, and definite increase in weight and of the number of erythrooytes is determinable. Four rabbits have died of nephritis.

Mal de Caderas.

Guinea-pigs (two). Animals are alive six and three-quarter months rom date of infection, and one month and a half after discontinuance of treatment. Parasites absent, blood non-infective. Bitch, two and a quarter months treated; no parasites seen, blood non-infective since twenty-eight days.

With five exceptions every animal has had one or more controls which were infected at the same time; the controls of T. gambiense, strain " $e$," surra, nagana, caderas, and Gambian horse parasites have all died in the usual time. It is therefore evident, from the great majority of the experiments, that the treatment of animals infected with trypanosomes with this preparation or in combination with the dye either arrests the disease, thus prolonging the life of the animals, or apparently cures them. This is especially the case in animals infected with the ordinary strain of T. gambiense, and even, though to a less extent, with the abnormally virulent, strain called " $e$."

A comparison of animals infected with the same strains, but treated according to Laveran's method with sodium arseniate has made me conclude that treatment with this aniline compound is in many ways superior to the ordinary arsenical treatment, on account of the quicker action of the drug on the parasite, the fact that the action seems to be prolonged, that large doses can be given without toxic symptoms and the entire absence from any tendency to cause sloughing.

In my opinion treatment is indicated of cases of trypanosomiasis in man with this drug in high doses administered intravenously and for a long period, pushing it to the maximal amount that the case can stand without headache and nausea, at the same time building up the patient in every way possible that will conduce to a lessening of the anaemia. In the European the combination of trypan red would probably be objectionable on account of the intense colouring of the tissues and secretions, but the native exhibits only a reddening of the conjunctivae and staining of the secretions. One of my cases of trypanosome fever had been under treatment with trypan red and arsenic for a short time before he returned to the Congo with favourable results-the parasites were longer absent from the peripheral circulation; his general blood condition was better; his temperature was almost normal.

I present the results of my experiments tentatively. I have used the term "apparently cured," as any one with an trypanosomes in animals knows how difficult it is to say that an animal is not infected. This is especially the case with the human parasite. In a previous paper I have recorded cases in which the blood has been negative for nearly a year in rats ${ }^{9}$ known to have been infected, and which at the expiration of that time showed parasites once more in their blood.

I do not believe that sodium arseniate alone will be found of great practical value, nor do I think atoxyl is a perfect preparation, from its toxic effects on canines and felines, but it is an advance on arsenious acid, and, if further efforts be made to produce a substance like trypan red, but less irritating

* The experiments with trypan-red treatment are reserved for fuller 
in action, the combination ought to be of service in the treatment of trypanosomiasis in man.

\section{REFERENCES.}

1 J. E. Dutton. Thompson-Yates Laboratories Report, 1902, vol. iv, Part II, p. 455. 2 Royal Bociety, Reports of the 8leeping Bickness Commission, 1903. 3 Laveran, Anil Inst Pasteur, t. X7i, p. 792. 4 E. J. Moore, zoncet, 1904, vol. 11, p. 15. S C. R. Chichester, Journ. Trop. Med., 1904 7 P. Ehrilch and K. Bhiga. Berl klin. Woch, March 4th and April th, 1904 8 H. Wendelstadt, Deut med. Woch. and B. F. Iinton, Lancet, 1904, vol. 1, No. 20, p. 1336 .

\section{A CASE OF CEREBRO-SPINAL MENINGITIS : LUMBAR PUNCTURE : RECOVERY.}

\section{By JAMES DONELAN, M.B.,}

Chevalier of the Crown of Italy; Physician, Italian Hospital, London.

IN view of the increasingly-frequent occurrence during the last few weeks of cases of cerebro-spinal meningitis the following instance seems worthy of record, especially in view of the immediate improvement that followed the surgical intervention.

The patient, an English architect, aged 50, who had met with reverses of fortune, was found comatose in his lodgings on October 25 th, 1904, and removed to the Holborn Infirmary, where in an interval of consciousness he was able to communicate with his friends, and was transferred to the Italian Hospital on Uctober 26th. From his own subsequent account he was in his usual good health until October 23rd, when he was seized with shivering, violent headache, and severe pain in his neck and back. He became unconscious, and must have been in that state for nearly two days before he was found. In the hospital he lay semicomatose, but roused when spoken to and complained of intense occipital pain extending along his neck and spine. The neck was rigidly extended owing to clonic spasm of the muscles, and there was some opisthotonos, though not in a marked degree. Kernig's sign was easily elicited both in the sitting and recumbent postures. The conjunctivae were suffused and the pupils widely dilated, but reacted to light and for accommodation. There was a petechial rash all over the body, especially on the lower extremities, while the skin generally was hyperaesthetic. He was perspiring moderately, and the sweat had the peculiar odour usually associated with typhus. There was some coryza, with muco-purulent discharge from the nostrils; no bacteriological examination of this secretion was, however, made. The bowels were constipated and the breath extremely foul, partly owing, no doubt, to the neglected state of his teeth and the existence of an extensive pyorrhoea alveolaris. The temperature on admission was $100.3^{\circ} \mathrm{F}$. and the pulse ro8. The blood count showed leucocytosis, 26,000 per c.mm. There were no pulmonary or other symptoms than those already given.

In view of the coryza, the case was at first regarded as one of influenzal cerebro-spinal meningitis; still, the possibility of it being a sporadic example of epidemic cerebro-spinal meningitis was considered, and the patient was isolated. He was given a calomel purge followed by an enema, and ice was applied to the head and along the spine. A mixture containing 20 minims of the ammoniated tincture of quinine and 5 minims of glycerine of carbolic acid in cinnamon water was given every four hours, and $20 \mathrm{gr}$. of bromide of potassium on the first two evenings. He was put on milk diet without alcohol.

On the 28th-that is, the fifth day from the seizure and within forty-eight hours of his admission-the leucocytosis having advanced to 36,000 , the temperature to $101^{\circ} \mathrm{F}$., and no improvement in the other symptoms having taken place but, on the contrary, a slight degree of aphasia having presented itself, it was decided to employ the lumbar puncture. This was performed under strict aseptic conditions by Mr. G. Lenthal Cheatle, surgeon to the hospital. The fluid, about $4 \mathrm{oz}$., rushed out with considerable force, and was slightly turbid when held to the light. Its withdrawal appeared to have an immediately beneficial effect, as the patient became quite conscious in a few hours, and the pain muscular spasm, and aphasia gradually disappeared in abou two days. On the day following the puncture the temperature fell to $99^{\circ}$, and he said he felt quite well but weak. Next day the temperature rose to $101.6^{\circ}$, but fell after the bowels were relieved by an enema.

In view of the great improvement in his condition it was not thought necessary to repeat the lumbar puncture; the cutaneous hyperaesthesia, however, persisted, bat disappearec at the end of the first week. He made an uninterrupted recovery in two weeks. He remained in the hospital, however, until he could be sent to a convalescent home, and was transferred to the care of the dental surgeon, Mr. McKay, who successfully treated his pyorrhoea.

A portion of the fluid removed through the lumbar puncture was examined by Mr. Cheatle at King's College, and was found to contain numerous polynuclear leucocytes, some of which enclosed the diplococcus intracellularis. A similar result was obtained from the specimen examined in the hospital laboratory by Dr. V. Pereira, Resident Medical Officer. It is perhaps to be regretted that a bacteriological examination was not made of the coryzal discharge or of the thick spongy incrustation on the teeth, as it nay have thrown some light on the path of invasion.

I think it may be fairly claimed that in this case its remarkably favourable termination was due to the early employment of the lumbar puncture. The therapeutic value of this procedure has been much disputed, but it has always had a strong advocate in Netter, who advises early and repeated lumbar puncture. The value of any remedy is proverbially enhanced by its speedy employment. Cito, bene; et recte, and the withdrawal of the fluid at the earliest possible moment seems to be imperatively called for in cerebro-spinal meningitis. Properly carried out it can do no harm, while it is of the ntmost importance to relieve tension on such essentially vital parts of the human organism. By so doing one has the best chance of avoiding pus formation, which will almost inevitably take place if the patient continues to live and the tension is not relieved.

Those who, like the writer, remember something of the Dublin epidemics of twenty years ago and over will recall the fact that patients who died on the second or third day had their meninges only congested, and the subarachnoid space more or less distended with a clear or slightly milky fluid. On the other hand, in those who did not die for a week or more the fluid varied from a yellowish creamy exudate to green pus. Had Quincke's suggestion been in vogue in those days and the tension been relieved by early lumbar pancture it is probable there would have been far fewer opportunities of viewing the last $t w 0$ stages of the inflammation post mortem. It may, perhaps, be said that patients have recovered without lumbar puncture, and that many died aiter several weeks in whom after death the fluid was found clear or nearly so. This, however, seems to point rather to greater resistance to probably a small initial dose of the diplococcus or one of less intense virulence. It cannot possibly be thought that the disease is in any sense self-limited, or that the adoption of measures to relieve tension as early as possible is other than imperatively necessary.

Weichselbaum, Fortschritte d. Med, 1887, v, pp. 573 to 620. Rolleston and Allingham, Lancet, 1899, i, p. 889. Osler, article in Encyclopaedia Medica. Osler, Lancet, 1899, i, p. 1699. Jaeger, Dert. med. Woch., 1899, July 20 th. Saundby, On the Etiology of Non-Tuberculous Mening tis. Birm. Med. Rev. 1900, Xlviij, 269 to 28r. Netter, Meningites Aiguees Non-Tubercuin Theth Century Practice Cipollina in Twentieth Century Practice. Clpollina e Maraglano, Del valoro diar

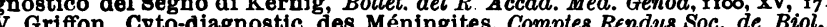
Paris liti, Ir to Pall von Meningitis Siderans, Miinch. med. 1201, 1, 459. Landau, Ein Fall von Meningitis Siderans, Miinch. mea. and Unusual Symptoms, BRITISH MEDICAL JOURNAL, 1901, 396.

\section{THE SODIUM SALTS OF THE COUMARIC ACIDS AS THERAPEUTIC AGENTS.}

BY GILBERT T. MORGAN, D.Sc.LoND., F.I.C., Royal College of Science, South Kensington.

TyE hypodermic application of sodium cinnamate in aqueous solutions (hetol) or dissolved in glycerine, as recommended by the author in the Lancet, July 12th, 1902, p. 66, still continues to receive considerable attention, and among the more recent developments of the treatment based on the use of this therapeutic agent may be mentioned the serum obtained by the firm of Kalle and Co., of Biebrich-am-Rhein, from tuberculous horses after repeated injections of the cinnamate (German patent No. 147,470).

Another point of interest bearing on the connexion between the chemical structure of cinnamic acid and its physiological action is the discovery that phenglpropiolic acid 